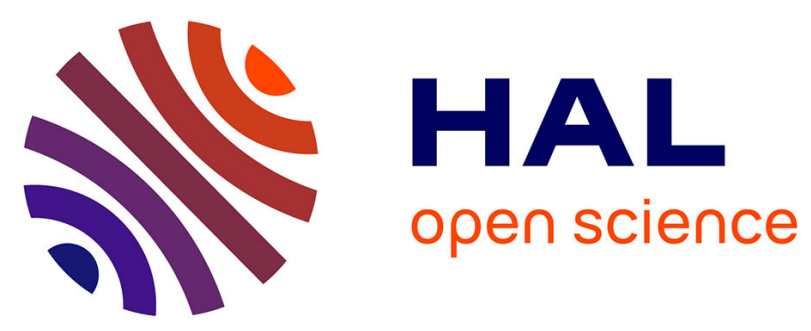

\title{
Sap flow measurement by a single thermal dissipation probe in transient regime: implementation of the method and test under field conditions
}

M. Masmoudi, Mahjoub, J. Lhomme, Ben Mechlia

\section{- To cite this version:}

M. Masmoudi, Mahjoub, J. Lhomme, Ben Mechlia. Sap flow measurement by a single thermal dissipation probe in transient regime: implementation of the method and test under field conditions. Annals of Forest Science, 2012, 69 (7), pp.773-781. 10.1007/s13595-012-0194-3 . hal-00930844

\section{HAL Id: hal-00930844 \\ https://hal.science/hal-00930844}

Submitted on 1 Jan 2012

HAL is a multi-disciplinary open access archive for the deposit and dissemination of scientific research documents, whether they are published or not. The documents may come from teaching and research institutions in France or abroad, or from public or private research centers.
L'archive ouverte pluridisciplinaire HAL, est destinée au dépôt et à la diffusion de documents scientifiques de niveau recherche, publiés ou non, émanant des établissements d'enseignement et de recherche français ou étrangers, des laboratoires publics ou privés. 


\title{
Sap flow measurement by a single thermal dissipation probe in transient regime: implementation of the method and test under field conditions
}

\author{
M. M. Masmoudi • I. Mahjoub • J. P. Lhomme • \\ N. Ben Mechlia
}

Received: 20 December 2011 / Accepted: 6 February 2012 /Published online: 24 February 2012

(C) INRA / Springer-Verlag France 2012

\begin{abstract}
- Context Recent works have showed the possibility of measuring sap flow density $(u)$ by a single heated probe using the transient regime just after the heating current is switched off. The method (SP-T) is based on a thermal index (I) which involves the temperature of the probe at three different times of the cooling kinetics (beginning, intermediate time, and end), the latter representing wood temperature at equilibrium. - Aims The present paper re-examines the method to better estimate wood temperature and to account for possible variations in probe-wood thermal properties.

- Methods After a recalibration of the method on an olive branch with a hydraulic bench, the SP-T method is used to measure sap flow on two olive trees under field conditions. - Results Estimated flow densities are compared to those obtained with the transient thermal dissipation-dual probe method (TTD-DP) of Do and Rocheteau.
\end{abstract}

Handling Editor: Erwin Dreyer

Contribution of the co-authors M.M. Masmoudi: experimental

work, modeling, data analysis, writing

I. Mahjoub: experimental work, data collection and analysis

J.P. Lhomme: modeling, writing

N. Ben Mechlia: writing, reviewing

M. M. Masmoudi $(\triangle) \cdot$ I. Mahjoub $\cdot$ N. Ben Mechlia

INAT, 43 Avenue Charles Nicolle,

1082 Tunis, Tunisia

e-mail: masmoudi.med@inat.agrinet.tn

I. Mahjoub

e-mail: imenemahjoub@hotmail.com

N. Ben Mechlia

e-mail: netij.benmechlia@iresa.agrinet.tn

J. P. Lhomme

IRD (UMR LISAH), Montpellier SupAgro,

2 place Viala,

34060 Montpellier, France

e-mail: Lhommejp@supagro.inra.fr
- Conclusions Obtained results show that: (1) wood reference temperature is better estimated when using the probe temperatures just before heating and at the end of the cooling phase; (2) calibration relationships based on a "normalized" thermal index $K_{t}=\left(I-I_{\mathrm{o}}\right) / I_{\mathrm{o}}$, where $I_{\mathrm{o}}$ represents the value of $I$ under zero flow conditions, better account for differences in the wood-probe thermal properties; (3) $I_{\mathrm{o}}$, taken as the lowest $I$ over a 24-h span, has shown consistent stability for a given probetree complex; (4) the SP-T method used in its normalized form appears to be in good agreement with the TTD-DP method.

Keywords Sap flow measurement . Thermal dissipation . Single probe $\cdot$ Transient regime $\cdot$ Olive tree

\section{Introduction}

During the last decades, various thermal methods have been developed to make sap flow measurement, which constitutes a practical way to estimate whole plant transpiration (Swanson 1994; Smith and Allen 1996; Cermak et al. 2004). The methods based on thermal dissipation were first developed by Granier $(1985,1987)$. Given that they are relatively simple and affordable in comparison to other methods, they have been widely used with different plants and under variable environments (e.g., Oliveras and Llorens 2001; O'Brien et al. 2004). The original design consists of two cylindrical needles radially inserted into plant stem: an upper probe, continuously heated by Joule effect, and an unheated probe approximately $10 \mathrm{~cm}$ below, used as temperature reference sensor. Thermocouples measure the temperature difference between the two sensors. The basis of the method is a calibration relationship linking sap flow density with the temperature difference, apparently valid for numerous types of plant and sensor (McCulloh et al. 2007). Two major limitations of the method, however, were 
reported. The first one is related to the natural thermal gradients often present in the wood, which affect the temperature difference (Do and Rocheteau 2002a). The second one is the need of zero flow conditions, which may not occur under certain circumstances (Lu et al. 2004).

Do and Rocheteau (2002b) developed a new approach called TTD-DP for Transient Thermal Dissipation-Dual Probe, which reduces the errors induced by natural thermal gradients. It is based on a thermal index defined from probes temperatures obtained by alternatively heating and cooling the upper probe. The original calibration relationship, first made on a sawdust column, was extended later to three tropical species and simplified to a linear relationship between sap flow and thermal index (Isarangkool $\mathrm{Na}$ Ayutthaya et al. 2010). Mahjoub et al. (2009) explored an approach which considers the use of a single heated probe and exploits the transient cooling regime just after the heating power is switched off. Calibrated on an olive tree branch, the method (hereafter called SP-T) appeared promising. However, it was not tested under field conditions, and there was some deficiency in the calibration relationship for low flow values. Do et al. (2011) also considered the use of a single probe and showed that, under stable reference temperature, the original thermal index of their dual probe method and the corresponding calibration relationship remained valid. When the reference temperature is not stable, the authors pointed out the necessity to linearly interpolate the cooled temperature. The proposed method (called TTD-SP) proved to yield similar sap flow estimates to those of the TTD-DP method on a young apple tree under rapid change of sapwood temperature.

In the present paper, the single probe method (SP-T) of Mahjoub et al. (2009) is revisited to discuss its practical implementation under field conditions and to propose some improvements. Physically founded, the SP-T thermal index involves the temperatures of the probe at three different times of the cooling phase: at the initial time of the kinetics $T(0)$, at an intermediate time $T\left(t_{\mathrm{i}}\right)$ and after $10 \mathrm{~min} T(600)$. This temperature $T$ (600) was supposed to approximate the mean temperature of the wood $\left(T_{\mathrm{w}}\right)$ during the period $\left[0-t_{\mathrm{i}}\right]$. The original calibration relationship implicitly assumed that the temperature of the wood remains constant during the cooling phase and that the equilibrium status is reached after $10 \mathrm{~min}$ (i.e., $600 \mathrm{~s}$ ). This assumption is questionable at low flow rates and a fortiori at zero flow. More time could be needed for the heated probe to reach complete thermal equilibrium with the wood. Additionally, under field conditions, rapid and large variations of external temperature may occur during the day leading to significant variations of wood temperature. This means that the temperature of the wood at the end of the cooling phase does not represent necessarily the one at the beginning (involved in the definition of the thermal index). The SP-T method also implicitly assumed the heat exchange coefficient of the probe-wood complex at zero flow to be a constant whatever the medium. This assumption is questionable: the coefficient may vary from tree to tree since it depends on the thermal properties of the probe, of the wood, and of the contact between them at the insertion point.

The present work examines some improvements of the method, focusing on a better estimation of $T_{\mathrm{w}}$ and on the way to account for possible variations in the heat exchange coefficient. These improvements are experimentally tested on a hydraulic bench and discussed in relation to the intermediate time $\left(t_{\mathrm{i}}\right)$. Then, the SP-T method is used to estimate sap flow densities of olive trees under field conditions and variable environmental and watering conditions. The estimated values are compared with those obtained by the dual probe (TTD-DP) method of Do and Rocheteau (2002b). Finally, we discuss the theoretical form of the SP-T calibration formula in comparison with the TTD-SP index of Do et al. (2011) and Granier's relationship.

\section{Materials and methods}

\subsection{The theoretical forms of the calibration relationship}

In the SP-T method, the temperature of the heating probe is measured with high frequency during the cooling phase (just after heating is switched off) and used to determine a thermal index (I) defined by the following formula (Mahjoub et al. 2009):

$I\left(t_{\mathrm{i}}\right)=\frac{1}{t_{\mathrm{i}}} \ln \left(\frac{T(0)-T_{\mathrm{w}}}{T\left(t_{\mathrm{i}}\right)-T_{\mathrm{w}}}\right)$

$T(0)$ is the temperature of the probe at the initial time of the kinetics ( $t=0$, when the heating current is switched off), $T\left(t_{\mathrm{i}}\right)$ is the temperature of the probe at an intermediate time $t_{\mathrm{i}}$ at the beginning of the cooling phase, $T_{\mathrm{w}}$ is the wood temperature approximated by the temperature of the probe at the end of the kinetics supposed to correspond to the thermal equilibrium between the probe and the wood. Based upon a simple heat transfer theory, a linear relationship between sap flow density $(u)$ and the thermal index $(I)$ was derived and experimentally calibrated on a hydraulic bench:

$u=a I\left(t_{\mathrm{i}}\right)+b$

Equation (2) is only valid for positive values of $u$, i.e., for values of $I$ greater than $-b / a$. The cut stem experiment on an olive branch reported in Mahjoub et al. (2009) showed that best results are obtained with $t_{\mathrm{i}}=20 \mathrm{~s}$, but intermediate times 
$t_{\mathrm{i}}$ within the range $10-60 \mathrm{~s}$ are also satisfactory. The coefficients $a$ and $b$ could be formulated as:

$a=C /\left(\alpha h_{0}\right)$

$b=-1 / \alpha$

where $C$ is the heat capacity of the probe, $h_{0}$ and $\alpha$ are parameters of the heat exchange coefficient $h$ which can be expressed as function of the sap flow $u$ as:

$h(u)=h_{0}\left(1+\alpha u^{\beta}\right)$

The linear calibration relationship, defined by Eq. (2), assumes power $\beta$ to be equal to 1 . As already mentioned in Mahjoub et al. (2009), the calibration relationship can also be written using a normalized index $K_{t}$ involving the thermal index $I_{\mathrm{o}}\left(t_{\mathrm{i}}\right)$ under zero flow conditions. It is expressed as:

$K_{t}\left(t_{\mathrm{i}}\right)=\frac{I\left(t_{\mathrm{i}}\right)-I_{\mathrm{o}}\left(t_{\mathrm{i}}\right)}{I_{\mathrm{o}}\left(t_{\mathrm{i}}\right)}$

Given that $I_{\mathrm{o}}\left(t_{\mathrm{i}}\right)=-b / a$, the calibration relationship can be rewritten as:

$u=\left(-b K_{t}\right)^{1 / \beta}=\left(K_{t} / \alpha\right)^{1 / \beta}$

This relationship has the same theoretical form as the original calibration relationship of Granier $(1985,1987)$ for the dual probe continuous thermal dissipation method: the coefficients $\alpha$ and $\beta$ have the same physical significance, but the definition of the normalized index $K_{t}$ is obviously different. The variable $I_{\mathrm{o}}$ plays the same role as $\Delta T_{\mathrm{M}}$ (maximum temperature difference between the two probes) in Granier's method or as $\Delta T_{\mathrm{o}}$ in the method of Do and Rocheteau (2002b). We will see that this normalized form of the calibration relationship is more adequate than Eq. (2) to account for medium variability.

\subsection{Calibration experiment on olive cut branch}

Calibration relationships were worked out from experimental data taken from Mahjoub et al. (2009) and from a new cut stem experiment carried out with an olive branch (Olea europaea L.). This experiment aimed at better calibrating the SP-T method for low values of sap flow and at performing a specific recalibration for olive specie of the dual probe (TTD-DP) method that will be used as a reference method under field conditions. The original calibration relationship of the TTD-DP method (Do and Rocheteau 2002b), reworked by Isarangkool Na Ayutthaya et al. (2010) on three tropical tree species with diffusive porous wood (Hevea brasiliensis, Mangifera indica, Citrus maxi$m a)$ is $u=12.95 K_{\text {a. }}$ Although the authors consider that the calibration relationship could be independent of the woody species, they recommend some sort of validation for different species of wood under different environmental conditions. The same hydraulic bench, already described in Mahjoub et al. (2009), was used to calibrate both methods. Pressurized water was injected at controlled flow rates into the olive tree branch equipped with measurement probes. The water flowing out was collected and weighed automatically by a digital balance ( $0.01 \mathrm{~g}$ precision).

In the TTD-DP method, the probe configuration is identical to that of Granier's method. It consists of two cylindrical needles radially inserted into the branch, the heated needle being placed $10 \mathrm{~cm}$ above the other (unheated). The temperature differences between the needles taken at the end of the heating period $\left(\Delta T_{\mathrm{on}}\right)$ and at the end of the cooling phase $\left(\Delta T_{\text {off }}\right)$ are used to calculate a thermal index $K_{a}=\left(\Delta T_{\mathrm{o}}-\Delta T\right) / \Delta T$, where $\Delta T=\Delta T_{\mathrm{on}}-\Delta T_{\text {off }}$ and $\Delta T_{\mathrm{o}}$ is the value of $\Delta T$ under zero flow conditions. Sap flow density $(u)$ is obtained using a calibration relationship with $K_{a}$. Following the recommendations by Isarangkool $\mathrm{Na}$ Ayutthaya et al. (2010), a cycle of 10 min heating and 20 min cooling was chosen, $\Delta T_{\mathrm{o}}$ was taken as the highest $\Delta T$ value and $\Delta T_{\text {off }}$ as the interpolated difference between the values before heating and after $10 \mathrm{~min}$ cooling. In order to have simultaneous measurement of sap flow by both methods using the same probes, the TTD-DP configuration was slightly modified: the two needles were used separately, cold junctions of both thermocouples were placed in an isothermal box, and reference temperature was measured by a precision NTC thermistor. This configuration allows simultaneous measurement of the temperatures of the heated and unheated needles. A data logger (Delta-T devices DL2e, Cambridge, UK) was used to control the heating-cooling sequence and to record the signals from the sensors and from the balance at $10 \mathrm{~s}$ time step. The branch diameter was approximately $5 \mathrm{~cm}$ and coloration of the wood by red-dye safranin showed that almost all the branch cross section was conductive. Consequently, the entire area of the cross section was used to derive water flow densities from the gravimetric flow values: they ranged from 0 to $51 \mathrm{dm}^{-2} \mathrm{~h}^{-1}$.

\subsection{Field experiment}

A field experiment on olive tree (O. europaea L.) was carried out during August and September 2008 in the experimental station of the "Institut National Agronomique de Tunisie". It is located in Mornag, close to the capital city of Tunisia $\left(36^{\circ} 41^{\prime} \mathrm{N}, 10^{\circ} 15^{\prime} \mathrm{E}\right)$. Average annual precipitation and reference evapotranspiration $\left(\mathrm{ET}_{0}\right)$ of the region are respectively around 450 and $1,200 \mathrm{~mm}$. Climatic factors such as global radiation and air temperature were measured at the experimental site. Olive trees were spaced away $6 \times$ 
$6 \mathrm{~m}$ and watered by drip irrigation. The system for sap flow measurement was installed on two trees (10 years old) conducted under differential irrigation regimes: tree 1 (trunk diameter $17 \mathrm{~cm}$ ) received twice the amount of irrigation water applied to tree 2 (trunk diameter $15 \mathrm{~cm}$ ). Sap flow densities were determined simultaneously by the single probe (SP-T) method and the dual probe (TTD-DP) method on a 30 -min basis during 16 days selected between August 28 and September 20.

Probe configuration was similar to the one used for the calibration relationships and described above. It consisted in using one heated needle and another one unheated, both being equipped with thermocouple. The same heated needle is used for both methods in order to reduce the errors associated with the variability of wood thermal properties and sap flow spatial heterogeneity. At the insertion point, the needles were thermally protected with polystyrene and radiation shields. Data loggers (Delta-T devices, Cambridge, UK) were used to control the heating process and to record the temperature of the probes as well as air temperature and solar radiation. Measurement cycles of $30 \mathrm{~min}$ were adopted with $10 \mathrm{~min}$ heating and $20 \mathrm{~min}$ cooling. Temperature of the probes was recorded every $10 \mathrm{~s}$. The thermal index (I) of the SP-T method was calculated using the temperatures of the heated probe at $t=0$ and at intermediate times $t_{\mathrm{i}}=10,20,30,40,50$, and $60 \mathrm{~s}$. Wood temperature $T_{\mathrm{w}}$ was estimated using different combinations of probe temperatures. After eliminating deficient measurements, the experimental data set resulted in 751 halfhourly values for tree 1 and 672 for tree 2 .

\subsection{Estimating wood temperature}

As already stressed, the correct estimation of wood temperature $T_{\mathrm{w}}$ is essential in the definition of the thermal index I $\left(t_{\mathrm{i}}\right)$ given by Eq. (1). In order to improve this estimation from temperature measurements of the heating probe, five estimators were tested. Putting $t=0$ at the beginning of the cooling period, $T_{\mathrm{w}}$ was successively calculated as: (1) the temperature of the probe after $10 \mathrm{~min}(600 \mathrm{~s})$ of cooling, as in the original formulation of the method: $T_{\mathrm{w} 1}=T(+600)$; (2) the mean between the temperature before the heating sequence $(-600 \mathrm{~s})$ and after $10 \mathrm{~min}$ of cooling $(+600 \mathrm{~s}): T_{\mathrm{w} 2}=$ $[T(-600)+T(+600)] / 2$; (3) the mean between $T(-600)$ and the temperature after $20 \mathrm{~min}$ of cooling $T(+1,200): T_{w 3}=[T$ $(-600)+T(+1,200)] / 2$; (4) the linearly interpolated value between $T(-600)$ and $T(+1,200): T_{w 4}=[2 T(-600)+T$ $(+1200)] / 3$; $(5)$ the linearly interpolated value between $T$ ( -600$)$ and the extrapolated value $T(+1,800)$ (assuming similar cooling trend during $1,200-1,800 \mathrm{~s}$ and $600-$ $1,200 \mathrm{~s}$ periods), calculated as $T_{\mathrm{w} 5}=[3 T(-600)+2 T$ $(+1,200)-T(+600)] / 4$.

\section{Results}

\subsection{Wood temperature estimates}

Using the data of the cut stem experiments, the five estimates of wood temperature detailed above were compared with the temperature of the wood $\left(T_{\mathrm{w}, \mathrm{r}}\right)$ effectively measured at the beginning of the cooling kinetics by the reference (unheated) probe inserted $10 \mathrm{~cm}$ below the heated one. The corresponding results are shown in Table 1 . The lowest values of root mean square errors (RMSE) and bias $(B)$ between estimated and measured values are obtained with $T_{\mathrm{w} 3}, T_{\mathrm{w} 4}$, and $T_{\mathrm{w} 5}$, which seem to be the best estimators of wood temperature. There is a small advantage for $T_{\mathrm{w} 5}$, which provides the lowest RMSE and $B$. Given the way this temperature is extrapolated, this advantage could be the indirect effect of the slow variation of lab temperature or of the fact that thermal equilibrium after $20 \mathrm{~min}$ is not reached yet (Do and Rocheteau 2002b). The small, but systematic, over-estimation could be attributed to thermal gradients within the wood.

Under field conditions, wood temperature follows the same variation as the external temperature. Figure 1 shows typical daily time courses of solar radiation, temperature of the air, and wood temperature measured by the reference probe (unheated) on tree 1. The range of variation of $T_{\mathrm{w}, \mathrm{r}}$ is much larger than the one observed during the cut stem experiment in the lab. As can be anticipated, under field conditions, the variation of temperatures is closely related to the corresponding variation of solar radiation. The time course of $T_{\mathrm{w}, \mathrm{r}}$ follows that of $T_{\mathrm{a}}$ with a lower amplitude and a time-lag of about $3 \mathrm{~h}$. For each tree, we compared the five estimates of wood temperature $\left(T_{\mathrm{w} 1}, T_{\mathrm{w} 2}, T_{\mathrm{w} 3}, T_{\mathrm{w} 4}\right.$, $T_{\mathrm{w} 5}$ ) with the temperature of the wood effectively measured at the beginning of the cooling kinetics by the unheated probe. Table 2 shows the results of this comparison. The discrepancy between estimated and measured values is larger than in the cut stem experiment (Table 1), certainly by default of thermal equilibrium. Despite the thermal gradients that may exist between the heated and unheated probes and the large variation of external temperature, $T_{\mathrm{w} 3}, T_{\mathrm{w} 4}$, and $T_{\mathrm{w} 5}$

Table 1 Root mean square error (RMSE), bias $(B)$, and determination coefficient $\left(R^{2}\right)$ between wood reference temperature $\left({ }^{\circ} \mathrm{C}\right)$ estimated by one of the five methods based on the temperature of the heated probe at different times of the cooling kinetics and the one $\left(T_{\mathrm{w}, \mathrm{r}}\right)$ measured by the unheated probe (results of the calibration experiment on hydraulic bench, $N=60$ )

\begin{tabular}{llllll}
\hline & $T_{\mathrm{w} 1}$ & $T_{\mathrm{w} 2}$ & $T_{\mathrm{w} 3}$ & $T_{\mathrm{w} 4}$ & $T_{\mathrm{w} 5}$ \\
\hline RMSE & 0.297 & 0.238 & 0.191 & 0.190 & 0.169 \\
$R^{2}$ & 0.818 & 0.893 & 0.914 & 0.920 & 0.928 \\
$B$ & 0.239 & 0.197 & 0.149 & 0.151 & 0.128 \\
\hline
\end{tabular}




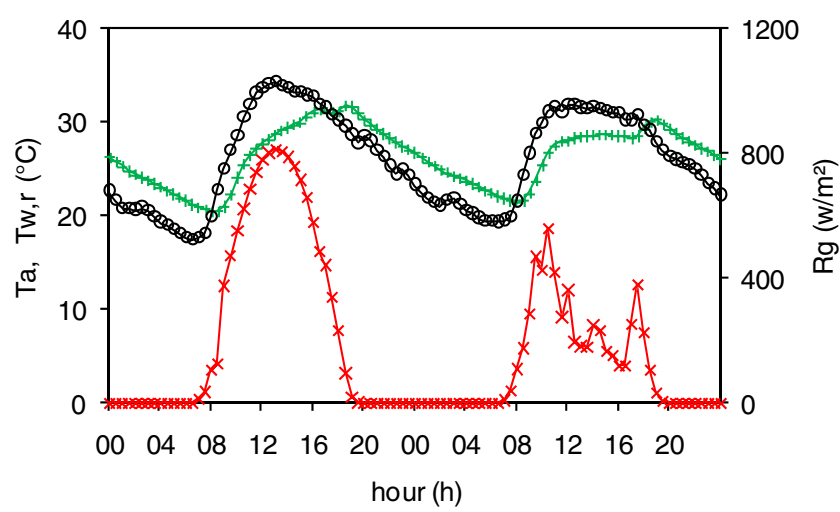

Fig. 1 Time variation of global radiation $\left(R_{\mathrm{g}}: \mathrm{x}\right)$, air temperature $\left(T_{a}\right.$ : o), and wood reference temperature $\left(T_{\mathrm{w}, \mathrm{r}}:+\right)$ during two contrasting consecutive days: $31 / 08 / 2008$ and $01 / 09 / 2008$

remain, nevertheless, the best estimators of $T_{\mathrm{w}, \mathrm{r}}$ (i.e., lowest RMSE and bias).

\subsection{Calibration relationships}

Following the recommendation of Isarangkool $\mathrm{Na}$ Ayutthaya et al. (2010), the dual probe method (TTD-DP) was recalibrated on hydraulic bench for olive tree. In Fig. 2, the water flow density $(u)$ gravimetrically measured during that experiment is plotted as a function of the dual probe thermal index $K_{a}$. The regression line (passing through zero) between $u$ and $K_{a}$ resulted in a specific coefficient of 14.42 for olive species with $R^{2}=0.96$. This value is slightly higher than the multi-species value (12.95) and is outside the confidence interval (12.39-13.56) given by Isarangkool $\mathrm{Na}$ Ayutthaya et al. (2010).

The data set from Mahjoub et al. (2009) was used together with that obtained with the new experiment in order to improve the calibration of the single probe method (SP-T) and to better account for low sap flow values. Table 3 shows the $R^{2}$ values of the linear regression lines between the gravimetrically measured flow density $(u)$ and the thermal

Table 2 Root mean square error (RMSE), bias $(B)$, and determination coefficient $\left(R^{2}\right)$ between wood temperature $\left({ }^{\circ} \mathrm{C}\right)$ estimated by $T_{\mathrm{w} 1}, T_{\mathrm{w} 2}$, $T_{\mathrm{w} 3}, T_{\mathrm{w} 4}$, and $T_{\mathrm{w} 5}$ and the reference one $\left(T_{\mathrm{w}, \mathrm{r}}\right)$ effectively measured by the unheated probe at the beginning of the cooling phase (results of the field experiment on two olive trees, $N=751$ for tree 1 and 672 for tree 2)

\begin{tabular}{llllllll}
\hline & Tree 1 & \multicolumn{5}{c}{ Tree 2 } \\
\cline { 2 - 4 } \cline { 6 - 7 }$T_{\mathrm{w}}$ & RMSE & $B$ & $R^{2}$ & & RMSE & $B$ & $R^{2}$ \\
\hline$T_{\mathrm{w} 1}$ & 0.786 & 0.647 & 0.990 & 1.132 & 0.787 & 0.964 \\
$T_{\mathrm{w} 2}$ & 0.775 & 0.557 & 0.984 & & 1.118 & 0.669 & 0.955 \\
$T_{\mathrm{w} 3}$ & 0.642 & 0.468 & 0.990 & & 0.958 & 0.551 & 0.964 \\
$T_{\mathrm{w} 4}$ & 0.687 & 0.468 & 0.986 & & 1.013 & 0.551 & 0.960 \\
$T_{\mathrm{w} 5}$ & 0.645 & 0.423 & 0.987 & 0.963 & 0.492 & 0.963 \\
\hline
\end{tabular}

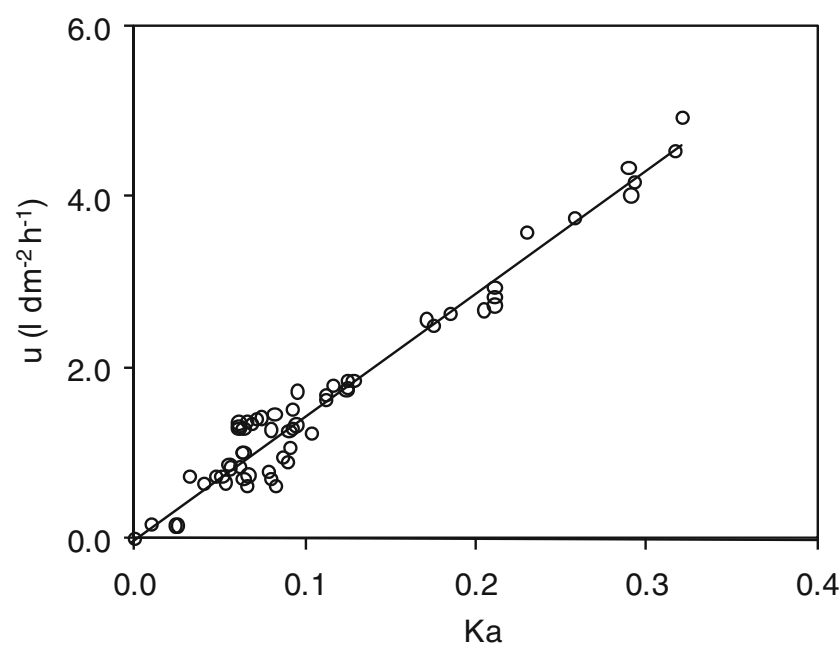

Fig. 2 Calibration relationship of the dual probe (TTD-DP) method obtained in the cut stem experiment: flow densities gravimetrically measured $(u)$ versus thermal index $K_{a}\left(u=14.42 K_{a}, R^{2}=0.96\right)$

index $(I)$ with flow densities ranging from 0 to $5 \mathrm{ldm}^{-2} \mathrm{~h}^{-1}$. The three best estimators of wood temperature $\left(T_{\mathrm{w}}\right)$ described above were used, and calculations were performed for intermediate time $t_{\mathrm{i}}$ varying from 10 to $60 \mathrm{~s}$. The corresponding $R^{2}$ values are slightly higher (above 0.96 ) than those previously obtained by Mahjoub et al. (2009). The root mean square errors (RMSE) between gravimetrically measured flows and those estimated by the calibration equations range between 0.21 and $0.24 \mathrm{ldm}^{-2} \mathrm{~h}^{-1}$.

The use of the normalized index $K_{\mathrm{t}}$ given by Eq. (6) requires the determination of $I_{\mathrm{o}}$ which is taken here as the value of the index $I$ extrapolated to zero flow conditions and calculated from the regression lines $u=a I\left(t_{\mathrm{i}}\right)+b$ (as $-b / a$ ). Table 4 gives the variation of $I_{\mathrm{o}}$ with $t_{\mathrm{i}}$, showing a decrease from about 0.067 to 0.022 when intermediate time $t_{\mathrm{i}}$ increases from 10 to $60 \mathrm{~s}$. Table 4 also shows the coefficient of the normalized calibration relationship $u=a_{\mathrm{n}} K_{\mathrm{t}}$ for different estimators of $T_{\mathrm{w}}$ and for $t_{\mathrm{i}}$ varying from 10 to $60 \mathrm{~s}$ : $a_{\mathrm{n}}=1 / \alpha$ in Eq. (7) assuming $\beta=1$. The corresponding $R^{2}$ values are identical to those shown in Table 3 . When using $T_{\mathrm{w} 4}$ as wood temperature estimator, the coefficient $a_{\mathrm{n}}$ varies from $7.6\left(t_{\mathrm{i}}=10 \mathrm{~s}\right)$ to $4.6\left(t_{\mathrm{i}}=60 \mathrm{~s}\right)$, while its value varies from 7.4 to 4.5 with $T_{\mathrm{w} 5}$ for the same $t_{\mathrm{i}}$. In Fig. 3, the regression lines between gravimetrically measured sap flow

Table 3 Determination coefficient $\left(R^{2}\right)$ of the regression lines between the observed water flow $(u)$ and the thermal index $I\left(t_{\mathrm{i}}\right)$ obtained using the three best estimators of wood temperature $\left(T_{\mathrm{w}}\right)$ and for $t_{\mathrm{i}}$ varying from 10 to $60 \mathrm{~s}$

\begin{tabular}{lllllll}
\hline$t_{\mathrm{i}}(\mathrm{s})$ & 10 & 20 & 30 & 40 & 50 & 60 \\
\hline$T_{\mathrm{w} 3}$ & 0.964 & 0.957 & 0.957 & 0.962 & 0.966 & 0.964 \\
$T_{\mathrm{w} 4}$ & 0.967 & 0.959 & 0.960 & 0.963 & 0.967 & 0.962 \\
$T_{\mathrm{w} 5}$ & 0.968 & 0.961 & 0.961 & 0.964 & 0.967 & 0.962 \\
\hline
\end{tabular}


Table 4 Thermal index under zero flow conditions $I_{\mathrm{o}}$ and coefficient $a_{\mathrm{n}}$ of the calibration relationship between the flow density $u$ and the normalized index $K_{\mathrm{t}}$ (regression line $u=a_{\mathrm{n}} K_{\mathrm{t}}$ with $\left.K_{\mathrm{t}}=\left(I-I_{\mathrm{o}}\right) / I_{\mathrm{o}}\right)$ (the results are shown for three wood temperature estimators and six intermediate times)

\begin{tabular}{llllllll}
\hline$t_{\mathrm{i}}(\mathrm{s})$ & & 10 & 20 & 30 & 40 & 50 & 60 \\
\hline$T_{\mathrm{w} 3}$ & $I_{\mathrm{o}}$ & 0.0673 & 0.0449 & 0.0349 & 0.0291 & 0.0251 & 0.0223 \\
& $a_{\mathrm{n}}$ & 7.6 & 6.2 & 5.5 & 5.2 & 4.8 & 4.7 \\
$T_{\mathrm{w} 4}$ & $I_{\mathrm{o}}$ & 0.0673 & 0.0449 & 0.0349 & 0.0290 & 0.0250 & 0.0223 \\
& $a_{n}$ & 7.6 & 6.1 & 5.4 & 5.1 & 4.8 & 4.6 \\
$T_{\mathrm{w} 5}$ & $I_{\mathrm{o}}$ & 0.0666 & 0.0444 & 0.0344 & 0.0286 & 0.0246 & 0.0219 \\
& $a_{\mathrm{n}}$ & 7.4 & 5.9 & 5.3 & 4.9 & 4.6 & 4.5 \\
\hline
\end{tabular}

density and the normalized index $K_{\mathrm{t}}$ are shown for $t_{\mathrm{i}}=20$, 40 , and $60 \mathrm{~s}$ using $T_{\mathrm{w} 4}$ as estimator of wood temperature. A slight discrepancy (or a lack of accuracy) seems to occur for low flow rates $\left(<0.5 \mathrm{ldm}^{-2} \mathrm{~h}^{-1}\right)$, but this should be checked by further experimental data.

\subsection{Field comparison}

Using the SP-T calibration relationships determined in the cut stem experiment, values of sap flow density were determined for both olive trees and compared to those obtained by the TTD-DP method for the 16 days of measurement. The value of $I_{\mathrm{o}}$ is supposed to be taken as the lowest value of the index $I$ during the 24 -h span. However, the $5 \%$ lowest values are systematically discarded in order to avoid errors associated with the noise that may affect electronic signals under field conditions. Table 5 gives the root mean square error (RMSE), bias $(B)$, and determination coefficient $\left(R^{2}\right)$ between the SP-T and TTD-DP methods for the three estimators of wood temperature $T_{\mathrm{w} 3}, T_{\mathrm{w} 4}$, and $T_{\mathrm{w} 5}$. Each value is the average corresponding to six intermediate times $t_{\mathrm{i}}$

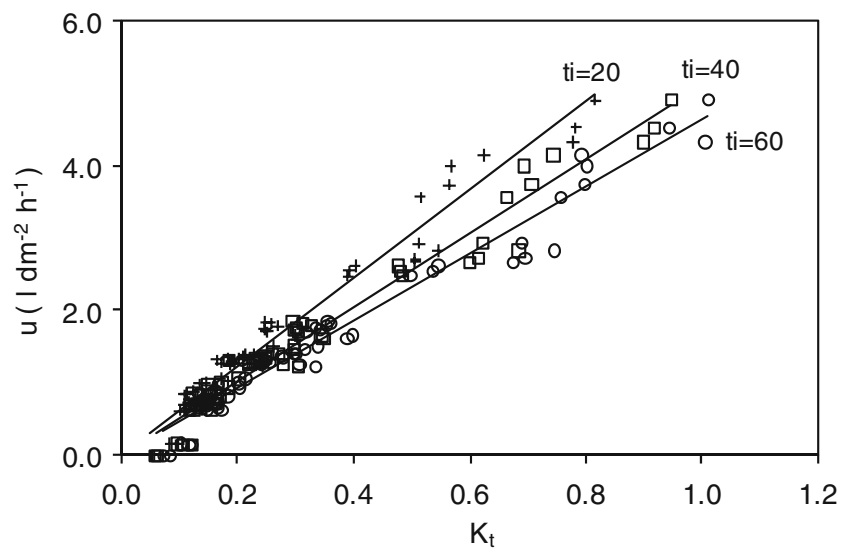

Fig. 3 Relationships between gravimetrically measured flow density $(u)$ and normalized index $K_{\mathrm{t}}$ for different values of the intermediate time $t_{\mathrm{i}}(\mathrm{s})$. Wood temperature $T_{\mathrm{w}}$ is estimated by $T_{\mathrm{w} 4}$
Table 5 Root mean square error (RMSE), bias $(B)$, and $R^{2}$ between sap flow densities $\left(1 \mathrm{dm}^{-2} \mathrm{~h}^{-1}\right)$ estimated by SP-T and TTD-DP methods for the two olive trees during the field experiment $(N=$ $1423)$ as a function of wood temperature $\left(T_{\mathrm{w}}\right)$ estimator: average values obtained by considering the six intermediate times $t_{\mathrm{i}}$ (from 10 to $60 \mathrm{~s}$ )

\begin{tabular}{lrrr}
\hline$T_{\mathrm{w}}$ estimator & \multicolumn{1}{c}{$T_{\mathrm{w} 3}$} & \multicolumn{1}{c}{$T_{\mathrm{w} 4}$} & \multicolumn{1}{c}{$T_{\mathrm{w} 5}$} \\
\hline RMSE & 0.183 & 0.149 & 0.148 \\
$B$ & +0.051 & -0.064 & -0.048 \\
$R^{2}$ & 0.976 & 0.982 & 0.979 \\
\hline
\end{tabular}

(from 10 to $60 \mathrm{~s}$ by $10 \mathrm{~s}$ ). The agreement between the two methods can be considered as very satisfactory. Figure 4 shows the comparison of sap flow densities estimated by the dual probe method with those obtained by the SP-T method using $T_{\mathrm{w} 4}$ as wood temperature estimator and $t_{\mathrm{i}}=40 \mathrm{~s}$ as intermediate time. Very similar results would have been obtained by using $T_{\mathrm{w} 3}$ or $T_{\mathrm{w} 5}$ and $t_{\mathrm{i}}$ varying from 10 to $60 \mathrm{~s}$. It appears that the SP-T method, when used adequately, provides sap flow estimates comparable to those given by the TTD-DP method. Figure 5 compares for two typical and contrasted days (August 31 and September 01) the time course of flow density in tree 1 estimated by the two methods together with global radiation. Sap flow density follows approximately the time course of solar radiation and reaches a maximum value of around $3.0 \mathrm{ldm}^{-2} \mathrm{~h}^{-1}$. Overall, the agreement between the two methods appears to be fairly good.

\section{Discussion}

4.1 The normalized form of the calibration relationship

The idea developed in this work is that, whereas the basic calibration relationship given by Eq. (2) is medium specific,

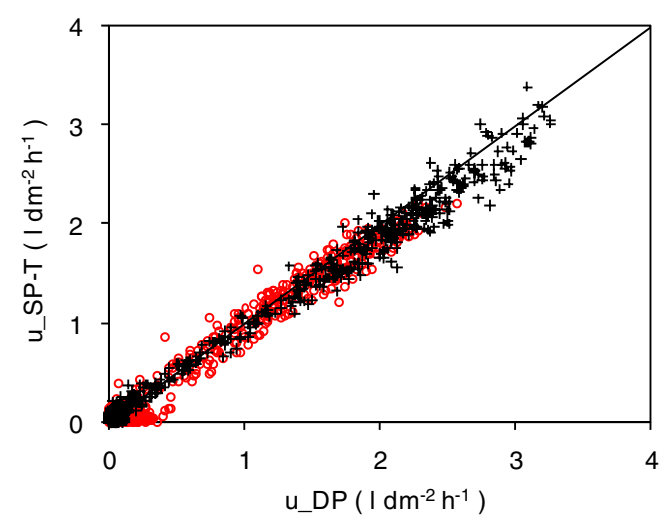

Fig. 4 Half-hourly values of sap flow density $(u)$ estimated by the SP$\mathrm{T}$ method (with $t_{\mathrm{i}}=40 \mathrm{~s}$ and $T_{\mathrm{w} 4}$ as estimator of wood temperature) versus those obtained with the TTD-DP method for well-irrigated $(+)$ and deficit-irrigated (o) olive trees 


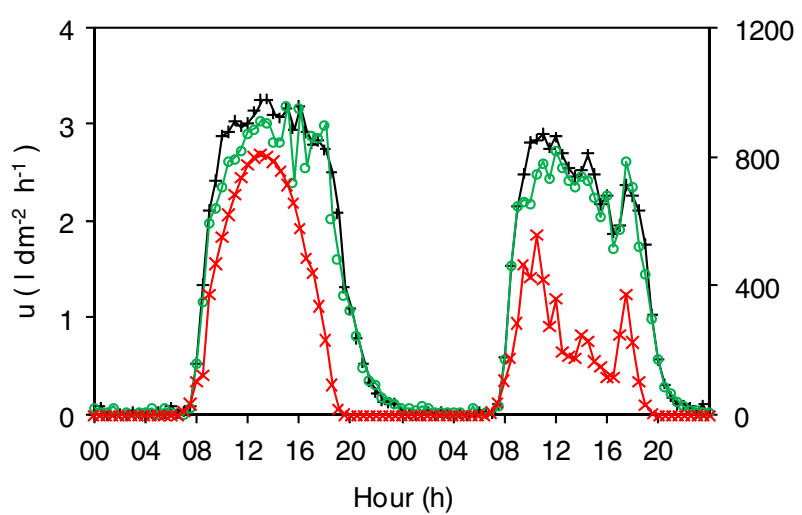

Fig. 5 Time course of half-hourly values of global radiation $R_{g}(\mathrm{x})$ and sap flow density $(u)$ estimated by the SP-T method (o) and TTD-DP method $(+)$ on olive tree 1 (well irrigated) during two contrasting and consecutive days (31/08/2008 and 01/09/2008)

the normalized form given by Eq. (7) is not and can be used in a systematic way with different trees and species. In this section, we present theoretical arguments supporting this idea. As a matter of fact, the normalized form has the advantage of accounting for some thermal properties of the probe-wood system through the index $I_{\mathrm{o}}$, allowing the calibration relationship to be extrapolated from one medium to another. Considering the equations developed above, (Eqs. (2) to (4)), we can notice that $I_{\mathrm{o}}\left(t_{\mathrm{i}}\right)=-b / a=h_{0} / C$, where $C$ is the heat capacity of the probe and $h_{0}$ the heat exchange coefficient of the probe when only heat conduction occurs $(u=0)$. Consequently, from a theoretical standpoint, $I_{\mathrm{o}}\left(t_{\mathrm{j}}\right)$ should depend only on probe characteristics and on the medium in which it is inserted. Whereas the capacity $C$ can be assumed to be constant for probes made with the same material and size, it is reasonable, on the other hand, to admit that $h_{0}$ depends on the type of wood (dense or light), on trunk geometry and on the thermal contact between the probe and the wood. Therefore, the index $I_{\mathrm{o}}\left(t_{\mathrm{i}}\right)$ should vary with the medium, but should be considered as approximately constant for a given medium-probe system.

Table 4, however, shows that for the olive branch of the cut stem experiment $I_{\mathrm{o}}\left(t_{\mathrm{j}}\right)$ decreases with increasing $t_{\mathrm{i}}$ : for instance, it goes from 0.067 to 0.022 when $t_{\mathrm{i}}$ varies from 10 to $60 \mathrm{~s}$ (using $\left.T_{\mathrm{w} 4}\right)$. This decrease of $I_{\mathrm{o}}\left(t_{\mathrm{i}}\right)$ is apparently in contradiction with the theoretical basis of the method. Nevertheless, we can notice that for values of the intermediate time higher than $30 \mathrm{~s}$, the value of $I_{\mathrm{o}}\left(t_{\mathrm{i}}\right)$ tends to be relatively constant compared to lower values (10-30 s). This would confirm that the method should be used with relatively high intermediate times $(40-60 \mathrm{~s})$. On the olive trees, it was found that $I_{\mathrm{o}}\left(t_{\mathrm{i}}\right)$ is effectively constant for a given tree and a given intermediate time $t_{\mathrm{i}}$ with a coefficient of variation always lower than $1 \%$ (results not shown) and second, that it decreases when $t_{\mathrm{i}}$ increases. Figure 6 shows how $I_{\mathrm{o}}\left(t_{\mathrm{i}}\right)$ decreases with $t_{\mathrm{i}}$ for trees 1 and 2: there is a slight difference

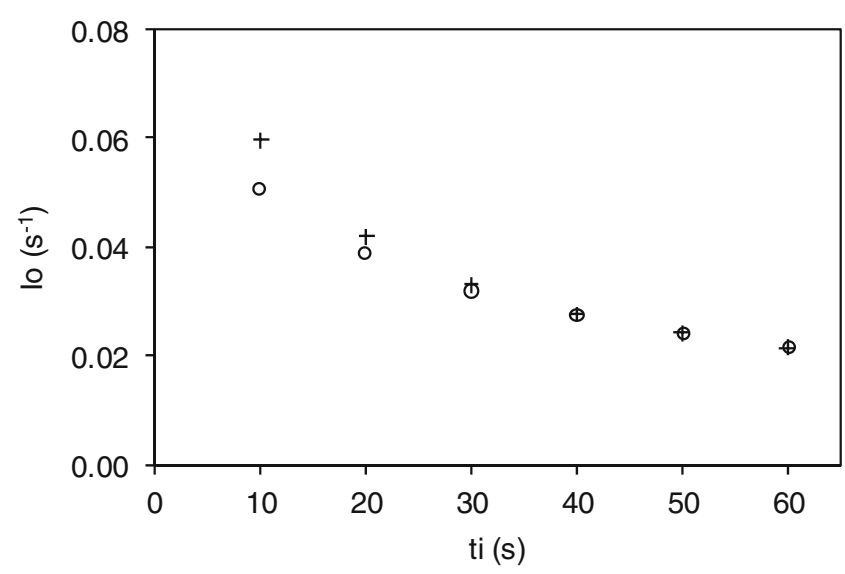

Fig. 6 Average values of the thermal index under zero flow conditions $\left(I_{\mathrm{o}}\right)$ for well-irrigated $(\mathrm{o})$ and deficit-irrigated $(+)$ olive trees observed during the experiment as a function of intermediate times $t_{\mathrm{i}}$ (from 10 to $60 \mathrm{~s})$

between the two trees for the low values of $t_{\mathrm{i}}$, but this difference almost disappears for $t_{\mathrm{i}}$ greater than $30 \mathrm{~s}$.

The decreasing behaviour of $I_{\mathrm{o}}\left(t_{\mathrm{i}}\right)$ with $t_{\mathrm{i}}$ is not completely odd and could have been anticipated by considering the characteristics of the kinetics detailed in (Mahjoub et al. 2009, "Wood temperature estimates" section, and Fig. 3). Under low flow conditions, the strict exponential phase of the kinetics is in fact delayed with respect to the switch off time (by more than $50 \mathrm{~s}$ for zero flow conditions), which can explain the departure from the theory when the intermediate time $t_{\mathrm{i}}$ is taken too short. It is clear also that the theoretical approach which supports the method is certainly only a mere approximation of much more complex phenomena of heat transfer within the wood (see Arkin 1989). Given that the wood medium is not infinite, but limited by the bark and the air; edge effects can partially invalidate the theory which sustains the method, mainly in the first seconds of the cooling kinetics. This kind of difficulty has already been noticed by Benet et al. (1977) with soil medium. The fact that the coefficient $\beta$ in the calibration relationship (Eq. (7)) was taken equal to 1, instead of the value of 0.81 obtained by Valancogne and Granier (1991), could be another reason for the observed discrepancy with the theory (i.e., the decline of $I_{0}$ with $t_{\mathrm{i}}$ ).

\subsection{Comparison with the TTD-SP method}

There is a clear similarity between the SP-T method discussed here and the TTD-SP method of Do et al. (2011) in their practical implementation: both use a single thermal dissipation probe with a cycle of heating and cooling. The fundamental difference, however, is in the nature of the thermal index. In the TTD-SP method, the index $K_{a}$ is essentially empirical and analogous (but not similar) to that of the dual probe (TTD-DP) method (see "Calibration experiment on olive cut branch" section). They are identical 
only in two cases: (1) if the wood reference temperature measured by the unheated sensor does not change over the cooling period; (2) if the "cooled" temperature (of the heated sensor) is interpolated and the reference temperature is also interpolated and varies linearly over a unit cycle (heating and cooling). The calibration relationships are also roughly identical, which supports the idea of a generic multi-species calibration for diffuse porous media (Isarangkool Na Ayutthaya et al. 2010). Overall, sap flow estimates do not differ between the two methods (TTD-DP and TTD-SP) provided that the cooled temperature is linearly interpolated.

On the other hand, the $K_{\mathrm{t}}$ index of the SP-T method is inferred from a physically founded analysis and has a different formulation. Besides, the "cooled" temperature of the heated probe $\left(T_{\mathrm{w}}\right)$ in the $K_{\mathrm{t}}$ index (Eq. (1)) has not exactly the same significance as in the $K_{a}$ index: in the SP-T method, the cooled temperature is supposed to represent exactly the reference wood temperature (at the time of the cooling onset) and should be estimated this way, whereas in the TTD-SP, it is not defined as such. Do et al. (2011) experimentally showed that there is a difference of up to $0.8-0.9^{\circ} \mathrm{C}$ between the two temperatures. For these reasons, the SP-T calibration relationship is quite different from that of the TTD-(DP or SP) method.

\subsection{Comparison with Granier's relationship}

As already mentioned ("The theoretical forms of the calibration relationship" section), the calibration relationships of the SP-T method and of Granier's steady state method are based upon the same theoretical equation (Eq. (7) with $\beta=$ 1): a thermal index $K$ multiplied by the coefficient $1 / \alpha$. The parameter $\alpha$ comes from the formulation of the heat exchange coefficient (Eq. (5)) and has the same physical significance in both methods. According to Granier (1985, 1987) and Valancogne and Granier (1991), the value of this parameter would be quasi universal and would not depend on the type of wood. Therefore, it is interesting to check whether the value given by Granier is close to the one obtained with the transient method.

In the original publication (Granier 1985), the experimental data points used for the calibration of the constant heating method (his Fig. 2) exhibit a linear trend, but it is a power function, with a higher $R^{2}(0.96)$, which was adjusted to the experimental points: $u=4.28 \mathrm{~K}^{1.23}$. In order to compare the two calibration relationships (constant and transient heating methods), the power function was transformed into a linear form, which yields approximately $u=4.3 \mathrm{~K}$. This value (4.3) of the theoretical coefficient $a_{n}=1 / \alpha$ is de facto very close to the values established with transient heating for large intermediate time $t_{\mathrm{i}}$, as shown in Table 4. The coefficient $a_{\mathrm{n}}$ varies from 4.9 to 4.5 when $t_{\mathrm{i}}$ varies from 40 to $60 \mathrm{~s}$ ( $T_{\mathrm{w} 5}$ being used as estimator of wood temperature). The fact that the coefficient is higher for $t_{\mathrm{i}}$ lower than $40 \mathrm{~s}$ should be examined in the light of the comments made about the variation of $I_{\mathrm{o}}$ : probe temperature experiences a delay in the strict exponential phase predicted by the theory, particularly for low flows. Nevertheless, the fact that the value of $a_{n}$ is very close to Granier's value (at least when the intermediate time $t_{\mathrm{i}}$ is relatively high) tends to give credit to the theoretical framework which supports the SP-T method. It also supports the idea that the "normalized" calibration relationships obtained under transient conditions on olive tree could be applied to other types of tree, but evidently this idea should be checked.

\section{Conclusion}

The SP-T method for sap flow measurement, first explored in Mahjoub et al. (2009) and developed here, has been made more operational and reliable by providing two major improvements to the method originally described. The first one concerns the correct estimation of wood temperature from the temperatures of the heated probe measured at different times of its kinetics. It has been shown that wood temperature is better estimated using an adequate combination of the temperatures of the probe before and after heating $\left(T_{\mathrm{w} 3}, T_{\mathrm{w} 4}\right.$, or $\left.T_{\mathrm{w} 5}\right)$ preferably with an intermediate time $t_{\mathrm{i}}$ greater than $30 \mathrm{~s}$. The second improvement consists in using the "normalized" form of the calibration relationship based upon the index given by Eq. (6). This form should be preferred to the original one because it accounts for the possible variation of thermal characteristics at the insertion point of the probe, which may differ from one tree to another. The index $I_{\mathrm{o}}\left(t_{\mathrm{i}}\right)$ corresponding to zero flow conditions should be determined in situ as the lowest value of the index over the day span after discarding $5 \%$ of index values. The single probe (SP-T) method for sap flow measurement was implemented in situ on olive trees and compared to the dual probe (TTD-DP) method of Do and Rocheteau (2002b). Following the rules above, a fairly good agreement was found between the two methods. It gives credit to this new method of sap flow measurement, which can be recommended for its operational use.

As the TTD-SP method of Do et al. (2011), the SP-T method presented here brings up some advantages with respect to the dual probe method. First, since only one probe is needed, it is cheaper and technically simpler, allowing one to increase the number of simultaneous measurements along the cross section of the tree. Second, the problems linked to natural thermal gradients within the wood are eliminated in principle. However, the method is not able to measure the direction of sap flow, which can be useful in studies with 
roots where reverse flows have been observed (Burgess et al. 2000).

In the future, the fact that the calibration relationship could be largely independent of the type of wood (as in Granier's method) should be checked on other mediums. Possible improvements of the SP-T method should also be considered: for instance, shortening the duration of the heating phase will allow shorter cooling, which would probably result in a better estimation of wood temperature and a reduction in measurement duration. Similarly, an intercomparison of the two single probe methods on different types of tree would be very useful.

\section{References}

Arkin H (1989) A thermal pulse method for the determination of sap flow velocity using a single probe: conceptual evaluation. J Agric Engineering Res 43:183-195

Benet JC, Pomade P, Hinzelin P (1977) Mesure de la conductivité thermique des sols par sonde cylindrique en régime transitoire. Etude de l'influence du mode de mise en place de la sonde. Matériaux et constructions 10:385-392

Burgess SSO, Adams MA, Bleby TM (2000) Measurement of sap flow in roots of woody plants: a commentary. Tree Physiol 20:909-913

Cermak J, Kucera J, Nadezhdina N (2004) Sap flow measurements with some thermodynamic methods, flow integration within trees and scaling up from samples trees to entire forest stands. Trees 18:529-546

Do F, Rocheteau A (2002a) A cyclic thermal dissipation system for measuring sap flow under high natural wood temperature gradients. I. Gradients and signals in the field. Tree Physiol 22:641648

Do F, Rocheteau A (2002b) A cyclic thermal dissipation system for measuring sap flow under high natural wood temperature gradients. II. Analysis in an artificial flow system. Tree Physiol 22:649-654

Do FC, Isarangkool Na Ayutthaya S, Rocheteau A (2011) Transient thermal dissipation method for xylem sap flow measurement: implementation with a single probe. Tree Physiol 31:369-380

Granier A (1985) Une nouvelle méthode pour la mesure du flux de sève brute dans le tronc des arbres. Ann Sci For 42:193-200

Granier A (1987) Evaluation of transpiration in a Douglas-fir stand by means of sap flow measurements. Tree Physiol 3:309-320

Isarangkool Na Ayutthaya S, Do FC, Pannengpetch K, Junjittakarn J, Maeght JL, Rocheteau A, Cochard H (2010) Transient thermal dissipation method of xylem sap flow measurement: multi-species calibration and field evaluation. Tree Physiol 30:139-148

Lu P, Urban L, Zaho P (2004) Granier's Thermal Dissipation Probe (TDP) method for measuring sap flow in trees: theory and practice. Acta Botanica Sinica 46:631-646

Mahjoub I, Masmoudi MM, Lhomme JP, Ben MN (2009) Sap flow measurement by a single thermal dissipation probe: exploring the transient regime. Ann For Sci 66:608

McCulloh KA, Winter K, Meinzer FC, Garcia M, Aranda J, Lachenbruch B (2007) A comparison of daily water use estimates derived from constant-heat sap-flow probe values and gravimetric measurements in pot-grown saplings. Tree Physiol 27:1355-1360

O'Brien JJ, Oberbauer SF, Clark DB (2004) Whole tree xylem sap flow responses to multiple environmental variables in a wet tropical forest. Plant Cell Environ 27:551-567

Oliveras I, Llorens P (2001) Medium-term sap flux monitoring in a Scots pine stand: analysis of the operability of the heat dissipation method for hydrological purposes. Tree Physiol 21:473-480

Smith DM, Allen SJ (1996) Measurement of sap flow in plant stems. J Exp Bot 47:1833-1844

Swanson RH (1994) Significant historical development in thermal methods for measuring sap flow in trees. Agric For Meteorol 1994:113-132

Valancogne C, Granier A (1991) Intérêt des méthodes thermiques de mesure du flux de sève pour l'étude du bilan hydrique des savanes. In: Soil Water Balance in the Sudano-Sahelian Zone (Proceedings of the Niamey Workshop, February 1991), IAHS Publ. No 199: 387-400. 